\title{
Stress Distribution of the Tibiofemoral Joint in a Healthy Versus Osteoarthritis Knee Model Using Image-Based Three-Dimensional Finite Element Analysis
}

\author{
Kulchamai Thienkarochanakul ${ }^{1} \cdot$ Akbar A. Javadi $^{1} \cdot$ Mohammad Akrami $^{1}$ ([) Joseph Robert Charnley ${ }^{1}$. \\ Abdelmalek Benattayallah ${ }^{2}$
}

Received: 27 November 2019 / Accepted: 20 April 2020 / Published online: 28 April 2020

(c) The Author(s) 2020

\begin{abstract}
Purpose Osteoarthritis (OA) is one of the most common pathological conditions to affect the human knee joint. In order to analyse the biomechanical causes and effects of OA, accessing the internal structures such as cartilage or the menisci directly is not possible. Therefore, computational models can be used to study the effects of OA on the stresses and strains in the joint and the susceptibility to deformations within the knee joint.

Methods In this study, a three-dimensional finite element model of a knee complex was constructed using MRI scans. Medical image processing software was used to create accurate geometries of bones, articular cartilages, menisci, patella, patella tendon and all the relevant ligaments. Finally, a 3D model of OA knee joint was created with a few changes to the cartilage. The cartilage was thinned, and the material properties were altered in order to simulate $\mathrm{OA}$ in the joint. 3D gait measurements were analysed to define loading and boundary conditions.

Results The developed model analysed the possibility of osteoarthritis. It was shown that the medial regions of cartilage layers and menisci in the knee joint sustain higher values of stress for OA conditions, while for the healthy knee, the stresses are more evenly distributed across the cartilage in the medial and lateral regions.

Conclusion The results suggest that any treatment for knee osteoarthritis should focus more on the medial region of the tibiofemoral cartilage in order not to cause degradation.
\end{abstract}

Keywords Finite element analysis $\cdot$ Knee $\cdot$ Biomechanics $\cdot$ Osteoarthritis

\section{Introduction}

The knee is one of the most complex joints in the human body and is responsible for significant load bearing during human locomotion. It is responsible for supporting the body and is integral to human locomotion. It facilitates the flexion and extension of the lower limb in the sagittal plane. The joint is composed of three main bones; tibia, fibula and femur and they are connected by four main ligaments which are anterior cruciate ligament (ACL), the medial collateral

Mohammad Akrami

m.akrami@exeter.ac.uk

1 Department of Engineering, College of Engineering, Mathematics, and Physical Sciences, University of Exeter, Exeter EX4 4QF, UK

2 Exeter MR Research Centre, University of Exeter, St. Luke's Campus, Exeter EX1 2LU, UK ligament (MCL), the lateral collateral ligament (LCL) and the posterior cruciate ligament (PCL). Cartilage layers provide the articulations within the joints, while the menisci absorb the shocks and bear the weight, providing a smooth locomotion pattern. An additional bone known as the patella (kneecap) makes up the joint but serves no mechanical function in load bearing. The cartilage and menisci sit inside a joint capsule composed of a fibrous tissue known as collagen, making the joint one of the most fragile in the body when also considering the weight-bearing forces to which it is exposed. The femur is the longest and most massive bone in the body, beginning at its proximal end at the hip joint and sweeping inwards towards the center of the body in the frontal plane as it extends towards the knee, so as to bring the knee joint more in line with the body's center of mass to improve balance. At the femur's distal end, it is met by the tibia, the body's second longest and heaviest bone. The tibia is the primary bone responsible for translating the 
weight of the body to the foot. The asymmetrical medial and lateral condyles protruding from the distal femur align with the equivalent plateau in the proximal tibia to form the main articulating structure of the knee. The final bone in the structure is the fibula, which forms the tibiofibular joint with the tibia. The fibula does not bear any weight, nor is it technically part of the knee joint; instead, it simply serves as a structure into which several muscles are inserted. The articulating surfaces at the distal and proximal ends of each bone in the knee joint are covered by a layer of cartilage. This is a porous, permeable, hydrated material that provides a near-frictionless surface against which joints can articulate as well as providing shock absorption. It is composed of four layers, each with different material structures and serving different functions. The innermost layer of the joint where the cartilages of opposing bones meet is responsible for lubrication in the joint. The calcified layer where cartilage meets bone anchors the cartilage, and the middle layers play roles in shock absorption. Between the cartilage layers of the femur and tibia lie the menisci, responsible for improving the congruency between the femoral condyles and the tibial plateau to increase the structural integrity of the joint. They consist of two semi-circular or C-shaped sections of fibrocartilage occupying an area between the femoral and tibial cartilages. The menisci share similar roles to those of the cartilage in assisting frictionless articulation and shock absorption. Whilst the cartilage is primarily responsible for protecting the bones and allowing articulation, the menisci play a larger role in distributing the loads transmitted through the femur and tibia, thus protecting the cartilage from degradation. They also prevent any lateral movement of the femur over the tibia. The menisci are attached to the tibial plateau via the coronary ligaments and the joint capsule. Four main ligaments connect the femur to the tibia: the anterior cruciate ligament (ACL), the medial collateral ligament (MCL), the lateral collateral ligament (LCL) and the posterior cruciate ligament (PCL). The LCL and MCL attach to the outside of the lateral and medial condyles and are responsible for keeping the femur and tibia in contact during flexion and extension. The ACL and PCL extend through the gaps in the femoral condyles. The ligaments also govern the positioning of the bones during articulation. If the cartilage layers degrade, the cartilage is worn away and after a while it is broken down causing knee osteoarthritis (OA) [1]. This is the most common form of OA, with the condition affecting $13 \%$ of people aged 55 to 64 and $70 \%$ of people aged 65 to 74 in the US [2,3]. In osteoarthritis, the cartilage is worn away and broken down, which causes the bones under the cartilage to rub together [4]. This, in turn, causes pain [5], stiffness [6], and a grating or grinding sensation (crepitus) [7] when the joint moves, swelling (either hard or soft) and restricted movement due to the affected joint. The etiology of OA is not fully understood; it most likely represents a number of different diseases with the same anatomical result. As such, there are many factors that can contribute to the onset of $\mathrm{OA}$, with biomechanical stress in particular appearing to be of significant influence. OA is characterized by degenerative changes to the morphology, composition and material properties of soft tissue within the joint. Whilst OA causes the wearing of the cartilage and menisci in the knee joint, the body's attempt to repair the damage causes more pain by the underlying bone grows outwards into the joint, forming bony horns called osteophytes [8]. These osteophytes affect the congruency of the joint, causing additional friction during movement and thus creating severe pain [8]. In such cases, OA can lead to the complete loss of the soft tissue between certain bone sites, causing them to articulate against each other and be worn down. Current treatments for OA depend on the severity of the case, with options ranging from total knee replacements to orthotic devices and knee braces.

In recent years, finite element modelling has been widely utilized to explain the biomechanics of the human knee and how this complex structure facilitates human movement. Although the finite element studies have improved our understanding of this vital structure [9-15], these computational models have not contributed to enhancing the remediation of knee conditions, e.g. in the case of knee OA, the biomechanics of which is still one of the unanswered research domains. Early computational models of the knee focus on more rudimentary properties of the joint. Blankevoort et al. generally examined articular contact properties of the knee using static analysis [16]. Pena et al. modelled the joint in order to better understand the combined role of the menisci and the ligaments in load transmission and stability of the knee [17]. Tarnita et al. addressed varus and valgus conditions, whereby the tibio-femoral angle was altered by $5^{\circ}$ in either direction in the frontal plane [8]. The same team conducted a similar study assessing more significant varus deformations and included behaviors under $0^{\circ}$ flexion with no varus or valgus angles [15]. More intricate biomechanical conditions have also been examined. In 2005, Pena et al. studied the effects of meniscal tears and meniscectomies on contact stress in the soft tissue [18]. This study was performed to assess any relationship meniscectomies may have to the onset of OA through increased cartilage degradation. Pathological conditions relating to the ligaments in the joint have also been investigated. Park et al. investigated the conditions under which the ACL becomes impinged against the femoral intercondylar notch to aid in the prevention and treatment of injuries associated with this phenomenon [19]. The biomechanical engineering studies have explained some clinical disorders [20-22], but osteoarthritis is one disorder that has not been fully investigated. Therefore, this study plans to provide a detailed three-dimensional model of the human knee and investigate the osteoarthritis effects on the 
tibiofemoral cartilage. This provides the ability to assess in vivo mechanical behavior of joints in the musculoskeletal system to greatly enhance our understanding of their functions and how pathological conditions occur. It is not possible to measure such behaviors in a non-invasive manner without the use of computational techniques such as finite element analysis (FEA). Such tools have been used to good effect in understanding how forces, stresses and strains act within joints. Therefore, a detailed bio-realistic model is developed and used to analyze the generation of the OA and compare its functional parameters against healthy subjects.

\section{Materials and Methods}

\subsection{Finite Element Modelling}

In this study, a complex bio-realistic model of a knee joint was constructed from medical MR images of a subject with a healthy knee (24 years old, with no history of lower limb injury or disease) in the supine position $\left(0^{\circ}\right.$ of knee flexion). The MRI scan data were collected on a $1.5 \mathrm{~T}$ Phillips Intera system using T1 3D Gradient Echo sequence (TR/ $\mathrm{TE}=57 \mathrm{~ms} / 21 \mathrm{~ms}$, flip angle $=3^{\circ}, 250$ very high spatial resolution slices with voxels size of $0.2 \times 0.2 \times 0.7 \mathrm{~mm}^{3}$ ). The images were segmented in order to define the boundaries of the bones and soft tissues using ScanIP software (Synopsys, Mountain View, USA). SolidWorks (Dassault Systèmes, SolidWorks Corp., USA) was used to apply necessary alterations for processing the boundary surfaces. The femur, tibia, fibula, and patella were the developed hard tissues, while the lateral and medial menisci and cartilage layers were segmented as shock absorbing soft tissues. The anterior cruciate ligament (ACL), posterior cruciate ligament (PCL), medial collateral ligament (MCL), and lateral collateral ligament (LCL) were also segmented as the three-dimensional structures of the knee ligaments that contribute to load transfer within the knee joint. In order to model the relative articulating movements within the joint, surface-to-surface contacts with finite sliding were assigned between the bones and their cartilage, and between the cartilages and meniscus while the insertion and origin of the ligaments were bonded to the bones. These contact properties were assigned to the model based on the literature $[25,26]$. The segmented parts were then exported and assembled to form the three-dimensional FE model of the knee joint using ABAQUS software (version 2016) (Dassault Systèmes, U.S.A) (Fig. 1). The model was initially constructed to examine the properties and function for the healthy knee conditions and it was then modified to represent $\mathrm{OA}$ conditions $[8,13]$. The material properties of the model were changed and the thickness of certain components of the model was altered to create the OA conditions, as OA occurs due to the thinning of the cartilage.

It is generally understood that OA mostly occurs on the medial side of the joint forming the varus condition, even though there is a possibility that OA can occur in the lateral zone forming the valgus condition [9]. This is due to the anatomical structure of the knee joint [23].

For the application of material properties for the OA condition, the cartilage and meniscus properties were changed
Fig. 1 Bio-CAD MRI based technique for modeling the human knee

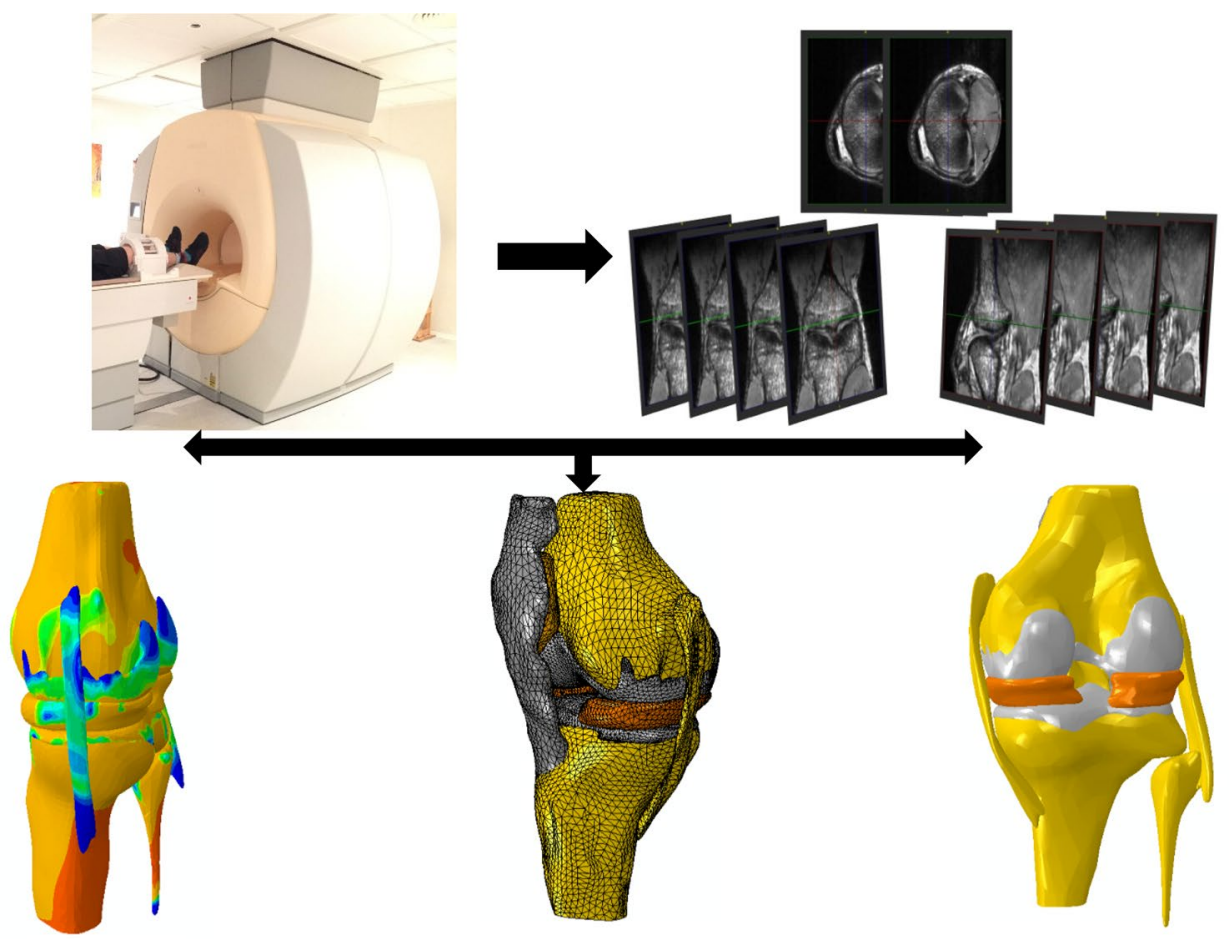


based on data from the literature $[12,25]$ (Table 1) and the thickness of the cartilage was reduced by $50 \%$. In this study, the analysis was performed based on the mid-stance phase of the gait where the leg is fully in contact with the floor and the knee joint is in full extension state. In order to replicate the mid-stance phase, the model was fixed at the distal end of the tibia. Zero displacements in $\mathrm{x}$ and $\mathrm{y}$ directions (on the horizontal plane) were assigned to the femur, preventing the bending or flexion of the joint.

A vertical load of $800 \mathrm{~N}$ was applied at the top of the femur, which corresponds to the force of full extension position in the gait cycle [24]. A displacement that allows offsetting in the $\mathrm{Z}$ axis and also rotation around the $\mathrm{Y}$ axis was assigned to the femur head. To identify the optimum mesh size, a mesh convergence analysis was conducted by continuously increasing the mesh density until the maximum deviations in the computed stresses became less than 5\% [25, 26] (Fig. 2). The geometries were discretised with average global mesh size of $2.5 \mathrm{~mm}$ for the bones, and $1.8 \mathrm{~mm}$ for the soft tissues. The simulation was done utilising a system with an Intel core-i5 6500 system, with 16 GB RAM.

\subsection{Experimental Measurements}

The kinematic variables for this gait study were captured using a motion capture system [24]. The system encompasses 3D camera scanners, a hub, markers and clusters. An active control hub (CodaHub) was utilized to integrate thirdparty data from the force platform (AMTI BP400600HF, Advanced Mechanical Technology Inc., USA) and run all four CX1 camera units whilst minimising real-time latency [24]. The three-dimensional gait measurement data were taken from the same subject who had volunteered for the MRI scans. The data from the motion capture experiments represented the 3D musculoskeletal model that was used to determine the maximum force input data (The Mathworks Inc., Cambridge, UK). In order to obtain accurate data, the markers were located in four main groups. The system included four 3D camera scanners, markers, two clusters, three driver boxes, micro-light gates, and force platform. In positioning the equipment, the two clusters were located on the thigh and shank, and each had four markers. The first driver box was located on the heel, at the 5th metatarsal, and the top of the foot with three markers on the box. The second driver box with four markers, was located on the calcaneus, on the 1st metatarsal, medial malleolus and lateral malleolus, and the third driver box with three markers was located on the greater trochanter, lateral knee and medial knee. The experimental results from the gait analysis were used in the FE model. In order to obtain a representative gait pattern, ten trials were recorded (Fig. 3).

\section{Results}

In knee OA, the medial compartment is usually first affected as the greatest compressive and rotational forces are transmitted through this compartment [10-12], as the medial femoral condyle is larger and slightly longer than the lateral condyle. The medial knee compartment is often associated with varus knee deformation, which consists of shifting of the mechanical axis and increased load bearing through the medial region, leading to increased severity and propagation of the disease.

Initially the model was used for analyzing the healthy knee model under the applied load of $800 \mathrm{~N}$. The maximum Von Mises stresses measured from the FEA were $2.76 \mathrm{MPa}$, 1.62 MPa, and 4.81 MPa on the femoral cartilage, tibial cartilage and menisci, respectively (Fig. 4). Also, the maximum predicted Von Mises stresses in the medial region were $0.96 \mathrm{MPa}, 1.53 \mathrm{MPa}$ and $1.59 \mathrm{MPa}$ on the femoral cartilage, tibial cartilage, and menisci, respectively.

These results obtained in the lateral region are slightly different from those presented by Tarnita et al. [8], who have reported maximum stresses of $2.41 \mathrm{MPa}, 2.17 \mathrm{MPa}$ and $2.12 \mathrm{MPa}$ on the femoral cartilage, tibial cartilage and menisci respectively, which are also taken at a lateral region of the joint (Table 2). One possible reason for this difference is the thickness of the menisci in this model compared to Tarnita et al. [8], which is different based on the subjects' anatomical differences. The stress distribution of each component is different. On the femoral
Table 1 Material properties of healthy and OA knee component, Element type and Number of Element that are assigned to the knee joint

\begin{tabular}{lllll}
\hline Part & $\begin{array}{l}\text { Young's modulus } \\
(\mathrm{E})[\mathrm{MPa}]\end{array}$ & $\begin{array}{l}\text { Poisson's ratio } \\
(\mathrm{v})\end{array}$ & Element type & No. of element \\
\hline Femur & 18,600 & 0.3 & Quadratic tetrahedral & 47,588 \\
Tibia & 12,500 & 0.3 & Quadratic tetrahedral & 35,160 \\
Healthy cartilage & 12 & 0.49 & Quadratic tetrahedral & 23,767 \\
Healthy menisci & 59 & 0.49 & Quadratic tetrahedral & 13,666 \\
Ligaments & 10 & 0.49 & Quadratic Tetrahedral & 11,236 \\
OA cartilage & 6 & 0.49 & Quadratic Tetrahedral & 35,129 \\
OA menisci & 29.5 & 0.49 & Quadratic Tetrahedral & 15,383 \\
\hline
\end{tabular}


Fig. 2 Mesh sensitivity study

Bone mesh sensitivity
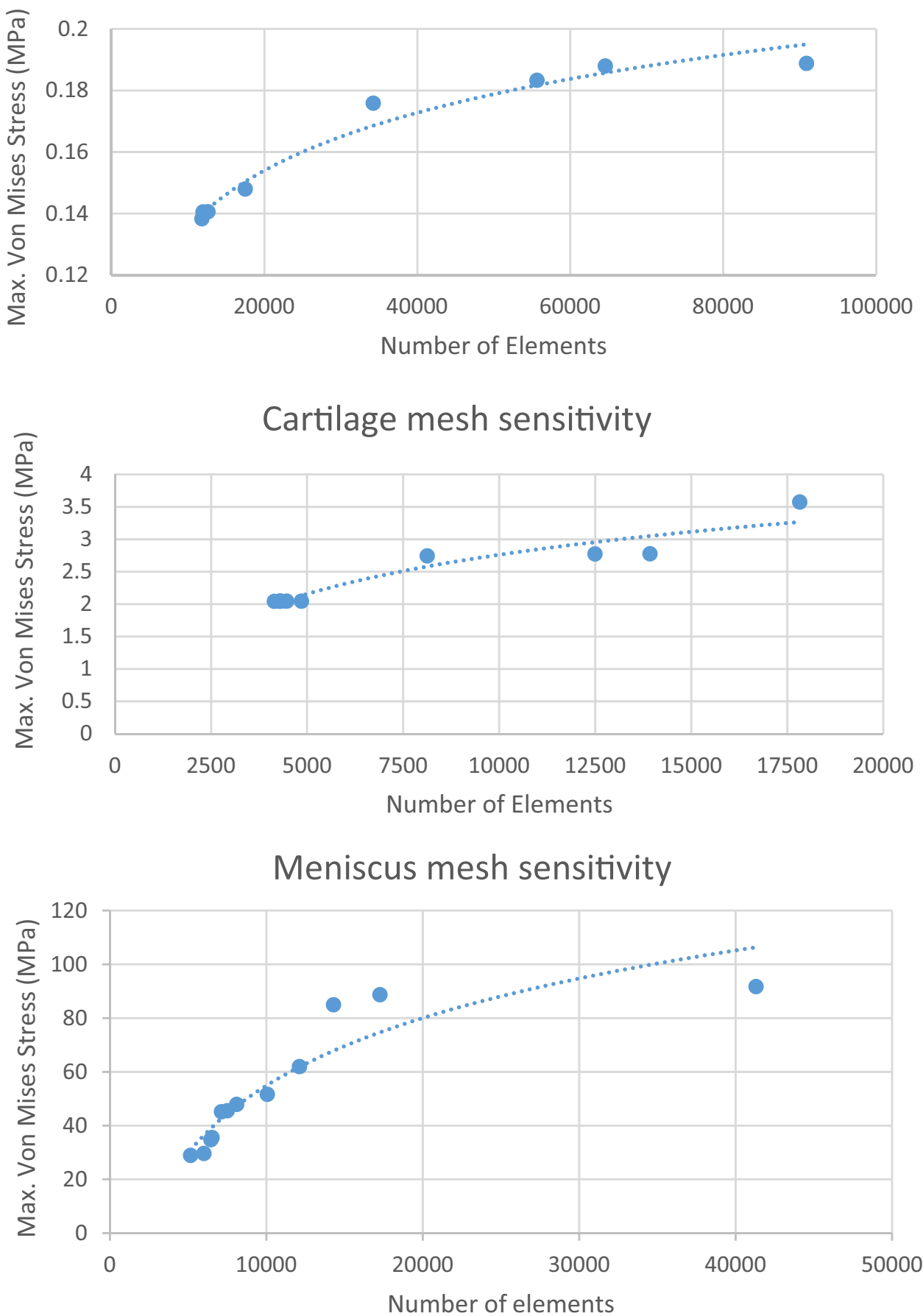

cartilage, the maximum stress occurred in the centre of the cartilage, while on the tibial cartilage the maximum stress was on the lateral-posterior side. On the other hand, on the menisci, the maximum stress occurred at the lateral-anterior side. For the loading of $1500 \mathrm{~N}$, the stress distribution and location of the maximum stress on each component changed. The predicted maximum stresses at $1500 \mathrm{~N}$ load were 2.796 MPa, 2.939 $\mathrm{MPa}$ and 7.441 $\mathrm{MPa}$ on the femoral cartilage, tibia cartilage and menisci respectively. In both cartilages, the maximum stress occurred on the lateralposterior side while on the meniscus, the maximum stress occurred on the lateral-anterior side. The results showed that for the OA, the disease generally occurs in the medial region of the knee joint [23]. Thus, the simulation was set up using different values for material properties of the cartilage and meniscus (Table 1). The model was modified 
Fig. 3 Ground reaction forces in $\mathrm{X}, \mathrm{Y}$ and $\mathrm{Z}$ during the gait pattern
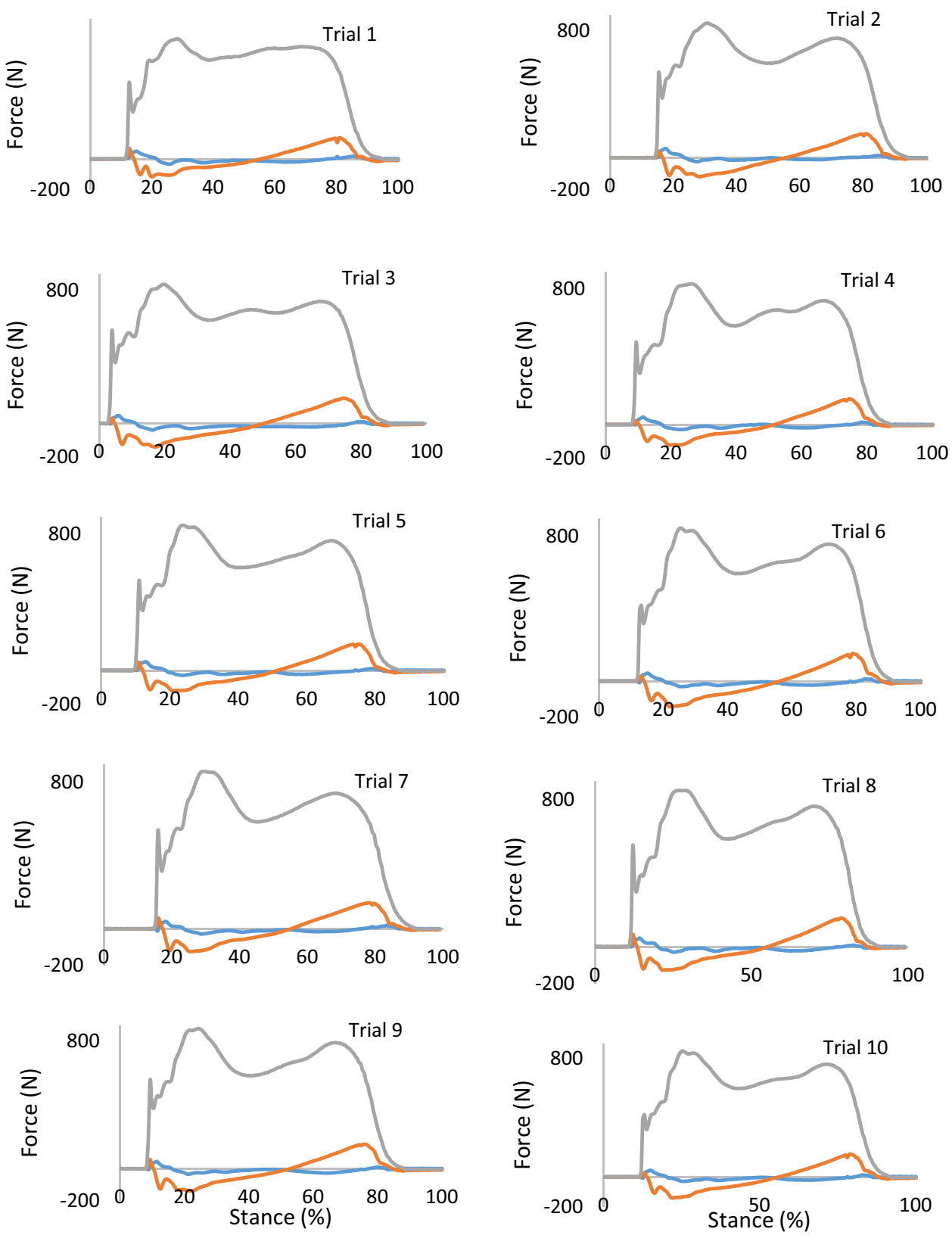

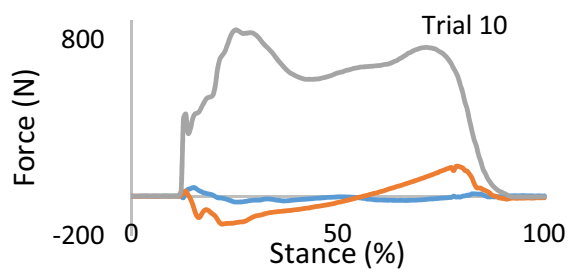

by reducing the thickness of the cartilage to replicate the worn cartilage in OA. The maximum predicted stresses in the medial region were $2.73,3.17$ and $2.82 \mathrm{MPa}$ on the femoral cartilage, tibial cartilage and menisci, respectively (Fig. 5). The stresses predicted in the lateral region of the $\mathrm{OA}$ analysis were $0.99,0.32$ and $0.61 \mathrm{MPa}$ on the femoral cartilage, tibial cartilage and menisci, respectively. When comparing the stress distribution in the lateral region of the OA knee against the result presented on the same region of the healthy knee, it is clearly shown that the stress within the OA knee joint has increased by more than $100 \%$, especially in the tibial cartilage and menisci, while on the femoral cartilage the difference in stress was $39 \%$ (Table 3).
On the other hand, the medial region of the healthy knee joint had a low stress distribution. The maximum predicted stresses in the medial region of the healthy knee joint were $0.96 \mathrm{MPa}, 1.53 \mathrm{MPa}$ and $1.59 \mathrm{MPa}$ on the femoral cartilage, tibial cartilage and menisci, respectively (Table 4). Comparison of the results shows that the OA knee joint sustained $96 \%$ more stress within the femoral cartilage, $70 \%$ more in the tibial cartilage and $56 \%$ more in the meniscus tissue, mainly in the medial region (Table 4). Vincent et al. [27] showed that the medial side of the knee is more susceptible for initiation and propagation of the Osteoarthritis. The most important parameter in this study, is the maximum von Mises stress, not the average stress, which causes pain and initiation of OA. As explained in Tables 3 and 4, the 


\section{Femoral Cartilage}

(a)

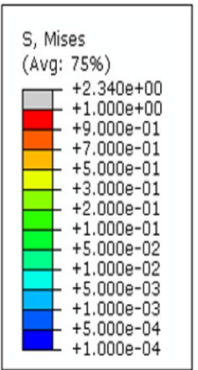

i.

즁

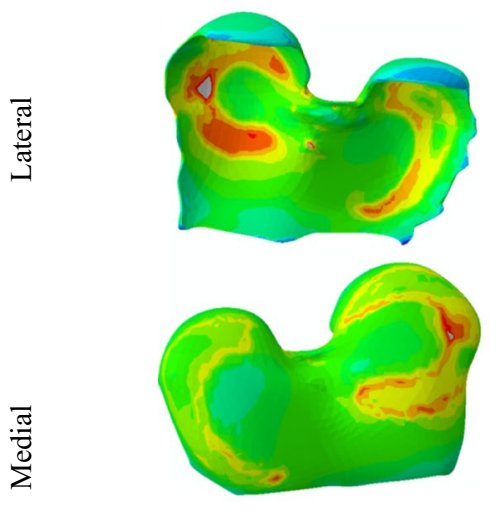

(b)

)
Tibia Cartilage

(c)

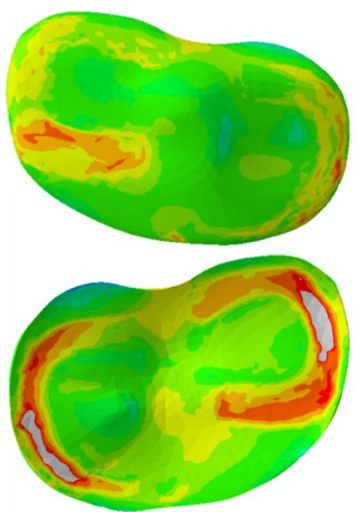

(d)
Menisci

(e)

Fig. 4 Simulated results of a healthy knee joint with $800 \mathrm{~N}$ loading a top view of femoral cartilage, $\mathbf{b}$ bottom view of femoral cartilage, $\mathbf{c}$ top view of tibia cartilage, $\mathbf{d}$ bottom view of tibia cartilage, $\mathbf{e}$ top view of menisci, $\mathbf{f}$ bottom view of menisci

Table 2 Comparison of the maximum von Mises stresses between the developed model with $800 \mathrm{~N}$ loading against Tarniţă et al. [8]

\begin{tabular}{lllc}
\hline Part component & $\begin{array}{l}\text { Tarniţă et al. } \\
{[8](\mathrm{MPa})}\end{array}$ & $\begin{array}{l}\text { Simulation result } \\
\text { on } 800 \mathrm{~N}(\mathrm{MPa})\end{array}$ & $\begin{array}{l}\text { difference in } \\
\text { stress }(\mathrm{MPa})\end{array}$ \\
\hline Femoral cartilage & 2.41 & 2.34 & 0.07 \\
Tibial cartilage & 2.17 & 1.582 & -0.588 \\
Menisci & 2.12 & 4.781 & 2.661 \\
\hline
\end{tabular}

maximum stress occurred in the medial part of the tibial cartilage following by menisci and femoral cartilage. The extracellular matrix of the cartilage and water within these structures facilitates the conditions to withstand significant loads, often multiple times of the body weight [28], and distribute it within its unique structure. But if shocks and stresses are maximised in a small region, this may cause pain because of the degradation of the joint cartilage [29].
Femoral Cartilage

$\mathrm{MPa}$

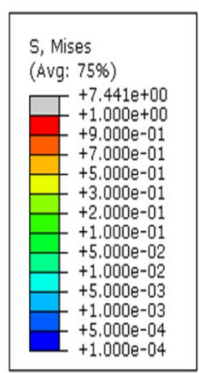

胥

(a)

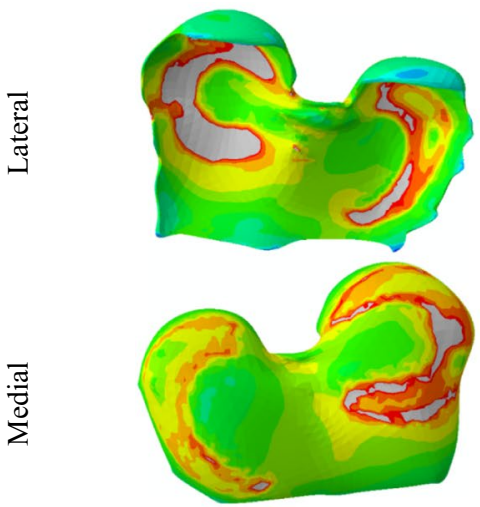

(b)
Tibial Cartilage

(c)

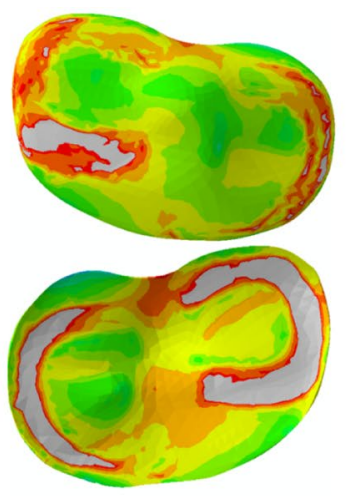

(d)
Menisci

(e)

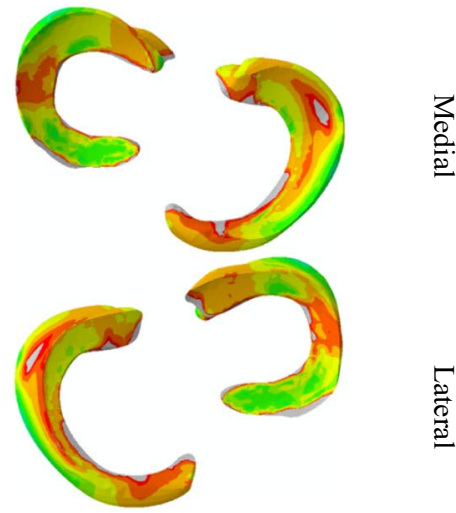

(f)

Fig. 5 Simulated results of an OA knee joint with $800 \mathrm{~N}$ loading a top view of femoral cartilage, $\mathbf{b}$ bottom view of femoral cartilage, $\mathbf{c}$ Top view of tibia cartilage, $\mathbf{d}$ bottom view of tibia cartilage, $\mathbf{e}$ top view of menisci, $\mathbf{f}$ bottom view of menisci 
Table 3 Comparison of the maximum von Mises stress of the OA and healthy knee models in the lateral region

\begin{tabular}{lllll}
\hline $\begin{array}{l}\text { Part compo- } \\
\text { nent }\end{array}$ & $\begin{array}{l}\text { Healthy knee } \\
\text { stress (MPa) }\end{array}$ & $\begin{array}{l}\text { OA knee } \\
\text { stress } \\
(\mathrm{MPa})\end{array}$ & $\begin{array}{l}\text { Difference in } \\
\text { stress (MPa) }\end{array}$ & $\begin{array}{l}\text { Percentage } \\
\text { deviation } \\
(\%)\end{array}$ \\
\hline $\begin{array}{c}\text { Femoral } \\
\text { cartilage }\end{array}$ & 1.466 & 0.986 & 0.48 & 39.15 \\
$\begin{array}{c}\text { Tibial carti- } \\
\text { lage }\end{array}$ & 1.582 & 0.318 & 1.264 & 133.05 \\
Menisci & 4.781 & 0.61 & 4.171 & 154.74 \\
\hline
\end{tabular}

\section{Discussion}

A three-dimensional finite element model was developed for a healthy patient. In the healthy knee joint, the stress distribution within the joint has shown that the maximum stresses occur in the lateral compartment. The load is gradually transferred from the femoral cartilage to the menisci and then to the tibial cartilage. The maximum stress predicted in the tibial cartilage is less than that in the femoral cartilage, which is due to the effects of the meniscus. As mentioned earlier, menisci play a vital role of shock absorption within the knee joint [30]. Thus, the stress that occurred in the tibial cartilage is less than that in the femoral cartilage.

Some modifications were made in the model, including changes in material properties as well as changes in thickness of the cartilage and menisci based on the subjects' anatomical differences. The results presented in this study show that the stresses within the OA knee joint are higher compared with the healthy knee joint in all components, including cartilages and menisci. The results of stress distribution and deformation of the model have shown that the lateral compartment is the most affected region, especially on the tibiofemoral cartilage. Comparison of the results of $\mathrm{OA}$ and healthy knee joints shows that the tibial cartilage is the most affected part of the joint. The maximum von Mises stress in the tibial cartilage increased by $85 \%$ compared to the healthy knee model. On the other hand, OA is a disease that causes deformation in the form of either varus or valgus conditions. In general, the medial region has the potential to be the most affected region, leading to varus deformity. Analysis was carried out on the OA model in which the material properties were modified, and the thickness of the cartilages was halved. Compared with the healthy model, the stresses in the OA knee model decreased in the lateral region and increased in the medial region. The tibial cartilage is the most affected cartilage in the OA with varus deformation.

\section{Conclusion}

A three-dimensional FE model of the human knee was developed and used to explain the biomechanics of osteoarthritis. Initially, a healthy model was developed and validated against the literature, while the input loading conditions were applied using the gait measurements. Then by some geometrical alterations and changes in material properties based on the literature, an OA knee model was developed and used for a comparative study. The results of the finite element analysis showed that the most susceptive region to the initiation of osteoarthritis is the medial region. It was also shown that while the healthy knee bears most of the weight in both regions equally, in the OA knee joint, the vast majority of the body weight is within the central and lateral regions of the tibiofemoral structure. The healthy knee model provided results with good proximity to those of another study [8], thus validating the accuracy of its results. The stresses seen in the menisci were greater than expected, but the stresses in the cartilages showed excellent similarity [8]. The model could therefore be modified to simulate $\mathrm{OA}$ in the joint. Whilst there are no published results that could be directly compared with the results obtained from this model, the increase in maximum stresses in the soft tissue is acceptable based on projections in the literature [31]. The FE modelling approach presented in this study explored the way in which the osteoarthritis is generated due to cartilage degeneration. The developed model provides a useful tool to study the consequences of OA conditions. However, the model still has certain limitations, including the need for further developments in the following aspects: (1) Patient-specific modelling of different bones and soft tissues would improve the accuracy of the numerical modelling for practical applications. (2) Bio-realistic loading conditions should be assigned based on individualised data by repeating and normalising such experiments, and (3) A meticulous validation of the FE simulation results for the
Table 4 Comparison of the maximum von Mises stress of the OA and healthy knee models in the medial region

\begin{tabular}{lllll}
\hline Part component & $\begin{array}{l}\text { Healthy knee stress } \\
(\mathrm{MPa})\end{array}$ & $\begin{array}{l}\text { The OA knee stress } \\
(\mathrm{MPa})\end{array}$ & $\begin{array}{l}\text { Difference in stress } \\
(\mathrm{MPa})\end{array}$ & $\begin{array}{l}\text { Percentage } \\
\text { deviation } \\
(\%)\end{array}$ \\
\hline Femoral cartilage & 0.956 & 2.728 & 1.772 & 96.2 \\
Tibial cartilage & 1.529 & 3.169 & 1.64 & 69.82 \\
Menisci & 1.585 & 2.824 & 1.239 & 56.2 \\
\hline
\end{tabular}


healthy and osteoarthritis subjects, within different genders and age groups, would increase the reliability of the finite element results and the sources of deviations.

Acknowledgements The authors would like to thank the "Simpleware software solutions" for providing the ScanIP software, especially Professor Philippe Young for all his support. Also, we thank the anonymous reviewers for their insightful comments and suggestions.

Funding None.

\section{Compliance with Ethical Standards}

Conflict of Interest The authors declare that they have no conflict of interest.

Ethical Approval Approved by the CEMPS ethics committee, University of Exeter, with the reference 281 number: eEMPS000040 v3.0.

Open Access This article is licensed under a Creative Commons Attribution 4.0 International License, which permits use, sharing, adaptation, distribution and reproduction in any medium or format, as long as you give appropriate credit to the original author(s) and the source, provide a link to the Creative Commons licence, and indicate if changes were made. The images or other third party material in this article are included in the article's Creative Commons licence, unless indicated otherwise in a credit line to the material. If material is not included in the article's Creative Commons licence and your intended use is not permitted by statutory regulation or exceeds the permitted use, you will need to obtain permission directly from the copyright holder. To view a copy of this licence, visit http://creativecommons.org/licenses/by/4.0/.

\section{References}

1. Zhang, W., Doherty, M., Peat, G., Bierma-Zeinstra, M. A., Arden, N. K., Bresnihan, B., et al. (2010). EULAR evidence-based recommendations for the diagnosis of knee osteoarthritis. Annals of the Rheumatic Diseases, 69, 483-489. https://doi.org/10.1136/ ard.2009.113100.

2. Chen, A., Gupte, C., Akhtar, K., Smith, P., \& Cobb, J. (2012). The global economic cost of osteoarthritis: How the UK compares. Arthritis, 2012, 698709. https://doi.org/10.1155/2012/698709.

3. Rosemann, T., Laux, G., \& Szecsenyi, J. (2007). Osteoarthritis: Quality of life, comorbidities, medication and health service utilization assessed in a large sample of primary care patients. Journal of Orthopaedic Surgery and Research, 2, 12. https://doi. org/10.1186/1749-799X-2-12.

4. Hashimoto, S., Ochs, R. L., Komiya, S., \& Lotz, M. (2004). Linkage of chondrocyte apoptosis and cartilage degradation in human osteoarthritis. Arthritis and Rheumatism, 41, 1632-1638. https:// doi.org/10.1002/1529-0131(199809)41:9\%3c1632:AID-ART14 \%3e3.0.CO;2-A.

5. Trueta, J. (1954). Osteoarthritis of the hip. Annals of the Royal College of Surgeons of England, 15, 174-192.

6. Dieppe, P. A., \& Lohmander, L. S. (2005). Pathogenesis and management of pain in osteoarthritis. Lancet, 365, 965-973. https:// doi.org/10.1016/S0140-6736(05)71086-2

7. Verzij1, N., DeGroot, J., Ben, Z. C., Braun-Benjamin, O., Maroudas, A., Bank, R. A., et al. (2002). Crosslinking by advanced glycation end products increases the stiffness of the collagen network in human articular cartilage: A possible mechanism through which age is a risk factor for osteoarthritis. Arthritis and Rheumatism, 46, 114-123.

8. Tarniţă, D., Catana, M., \& Tarnita, D. N. (2014). Modeling and finite element analysis of the human knee joint affected by osteoarthritis. Key Engineering Materials, 601, 147-150. https://doi. org/10.4028/www.scientific.net/KEM.601.147.

9. Schiphof, D., van Middelkoop, M., de Klerk, B. M., Oei, E. H. G., Hofman, A., Koes, B. W., et al. (2014). Crepitus is a first indication of patellofemoral osteoarthritis (and not of tibiofemoral osteoarthritis). Osteoarthritis and Cartilage, 22, 631-638. https ://doi.org/10.1016/J.JOCA.2014.02.008.

10. Bennell, K. L., Bowles, K.-A., Wang, Y., Cicuttini, F., DaviesTuck, M., \& Hinman, R. S. (2011). Higher dynamic medial knee load predicts greater cartilage loss over 12 months in medial knee osteoarthritis. Annals of the Rheumatic Diseases, 70, 1770-1774.

11. Nha, K. W., Dorj, A., Feng, J., Shin, J. H., Kim, J. I., Kwon, J. H., et al. (2013). Application of computational lower extremity model to investigate different muscle activities and joint force patterns in knee osteoarthritis patients during walking. Computational and Mathematical Methods in Medicine, 2013, 314280. https://doi. org/10.1155/2013/314280.

12. Mootanah, R., Imhauser, C. W., Reisse, F., Carpanen, D., Walker, R. W., Koff, M. F., et al. (2014). Development and validation of a computational model of the knee joint for the evaluation of surgical treatments for osteoarthritis. Computer Methods in Biomechanics and Biomedical Engineering, 17, 1502-1517. https:// doi.org/10.1080/10255842.2014.899588.

13. Cartana, M., Tarnita, D., \& Tarnita, D. (2013). Modelling, simulation and optimization of a human knee orthotic device. Applied Mechanics and Materials, 371, 549-553.

14. Sandell, L. J., \& Aigner, T. (2001). Articular cartilage and changes in Arthritis: Cell biology of osteoarthritis. Arthritis Research \& Therapy, 3, 107. https://doi.org/10.1186/ar148.

15. Loeser, R. F., Collins, J. A., \& Diekman, B. O. (2016). Ageing and the pathogenesis of osteoarthritis. Nature Reviews Rheumatology, 12,412 .

16. Blankevoort, L., Kuiper, J. H., Huiskes, R., \& Grootenboer, H. J. (1991). Articular contact in a three-dimensional model of the knee. Journal of Biomechanics, 24, 1019-1031. https://doi. org/10.1016/0021-9290(91)90019-J.

17. Peña, E., Calvo, B., Martínez, M. A., \& Doblaré, M. (2006). A three-dimensional finite element analysis of the combined behavior of ligaments and menisci in the healthy human knee joint. Journal of Biomechanics, 39, 1686-1701. https://doi. org/10.1016/j.jbiomech.2005.04.030.

18. Peña, E., Calvo, B., Martínez, M. A., Palanca, D., \& Doblaré, M. (2005). Finite element analysis of the effect of meniscal tears and meniscectomies on human knee biomechanics. Clinical Biomechanics, 20, 498-507. https://doi.org/10.1016/j.clinbiomec h.2005.01.009.

19. Park, H.-S., Ahn, C., Fung, D. T., Ren, Y., \& Zhang, L.-Q. (2010). A knee-specific finite element analysis of the human anterior cruciate ligament impingement against the femoral intercondylar notch. Journal of Biomechanics, 43, 2039-2042. https://doi. org/10.1016/j.jbiomech.2010.03.015.

20. Bendjaballah, M. Z., Shirazi-Adl, A., \& Zukor, D. J. (1995). Biomechanics of the human knee joint in compression: Reconstruction, mesh generation and finite element analysis. The Knee, 2, 69-79. https://doi.org/10.1016/0968-0160(95)00018-K.

21. Moglo, K. E., \& Shirazi-Adl, A. (2003). On the coupling between anterior and posterior cruciate ligaments, and knee joint response under anterior femoral drawer in flexion: A finite element study. Clinical Biomechanics, 18, 751-759. https://doi.org/10.1016/ S0268-0033(03)00140-2. 
22. Sawatari, T., Tsumura, H., Iesaka, K., Furushiro, Y., \& Torisu, T. (2006). Three-dimensional finite element analysis of unicompartmental knee arthroplasty-the influence of tibial component inclination. Journal of Orthopaedic Research, 23, 549-554. https ://doi.org/10.1016/j.orthres.2004.06.007.

23. Vincent, K. R., Conrad, B. P., Fregly, B. J., \& Vincent, H. K. (2012). The Pathophysiology of osteoarthritis: A mechanical perspective on the knee joint. $P M \& R, 4, \mathrm{~S} 3-9$. https://doi. org/10.1016/J.PMRJ.2012.01.020.

24. Peña, E., Martínez, M. A., Calvo, B., Palanca, D., \& Doblaré, M. (2005). A finite element simulation of the effect of graft stiffness and graft tensioning in ACL reconstruction. Clinical Biomechanics, 20, 636-644. https://doi.org/10.1016/J.CLINBIOMEC H.2004.07.014.

25. Akrami, M., Qian, Z., Zou, Z., Howard, D., Nester, C. J., \& Ren, L. (2018). Subject-specific finite element modelling of the human foot complex during walking: sensitivity analysis of material properties, boundary and loading conditions. Biomechanics and Modeling in Mechanobiology, 17, 559-576. https://doi.org/10.1007/ s10237-017-0978-3.

26. Akrami, M., Craig, K., Dibaj, M., Javadi, A. A., \& Benattayallah, A. (2018). A three-dimensional finite element analysis of the human hip. Journal of Medical Engineering \& Technology, 42, 546-552. https://doi.org/10.1080/03091902.2019.1576795.

27. Vincent, K. R., Conrad, B. P., Fregly, B. J., \& Vincent, H. K. (2012). The pathophysiology of osteoarthritis: A mechanical perspective on the knee joint. $P M \& R, 4(5)$, S3-S9. https://doi. org/10.1016/j.pmrj.2012.01.020.

28. Sophia Fox, A. J., Bedi, A., \& Rodeo, S. A. (2009). The basic science of articular cartilage: Structure, composition, and function. Journal of Sport and Health, 1, 461-468. https://doi. org/10.1177/1941738109350438.

29. Zafar, A. Q., Zamani, R., \& Akrami, M. (2019). The effectiveness of foot orthoses in the treatment of medial knee osteoarthritis: A systematic review. Gait \& Posture, 76, 238-251. https://doi. org/10.1016/J.Gaitpost.2019.12.016.

30. Bendjaballah, M. Z., Shirazi-Adl, A., \& Zukor, D. J. (1997). Finite element analysis of human knee joint in varus-valgus. Clinical Biomechanics, 12, 139-148. https://doi.org/10.1016/S0268 -0033(97)00072-7.

31. Donahue, T. L., Hull, M. L., \& Rashid, M. M. (2002). A finite element model of the human knee joint for the study of tibio-femoral contact. Journal of Biomechanical Engineering, 124, 273. https:// doi.org/10.1115/1.1470171. 